\title{
Open Environmental Platforms: Top-Level Components and Relevant Standards
}

\author{
Dumitru Roman ${ }^{1}$, Sven Schade $^{2}$, and Arne J. Berre ${ }^{1}$ \\ ${ }^{1}$ SINTEF, Oslo, Norway \\ \{dumitru.roman, arne.j.berre\} asintef.no \\ ${ }^{2}$ Institute for Environment and Sustainability, \\ Joint Research Centre of the European Commission, Ispra, Italy \\ sven.schade@jrc.ec. europa.eu
}

\begin{abstract}
We present our ideas of an open Information and Communication Technology (ICT) platform for monitoring, mapping and managing our environment. The envisioned solution bridges the gap between the Internet of Things, Content and Services, and highly specific applications, such as oil spill detection or marine monitoring. On the one hand, this environmental platform should be open to new technologies; on the other hand, it has to provide open standard interfaces to various application domains. We identify core components, standards, and needs for new standard development in ICT for environment. We briefly outline how our past and present activities contribute to the development of the desired open environmental platform. Future implementations shall contribute to sustainable developments in the environmental domain.
\end{abstract}

Keywords: open environmental platform, infrastructure, architecture, standards.

\section{Introduction}

The Shared Environmental Information System (SEIS) ${ }^{1}$ is one of three recent major initiatives along with the INSPIRE Directive (European Parliament and Council 2007) and the Global Monitoring for Environment and Security (GMES) ${ }^{2}$ undertaken by Europe to collect and share environmental information for the benefit of the global society. ICT have an essential role to play in the context of environmental information systems as they provide the necessary support in terms of tools, systems and protocols to establish a dynamic environmental space of collaboration in a more and more sophisticated digital world. Core challenges are not only related to providing seamless environmental data access to public authorities, businesses and the public at large, but also to allowing for interoperable environmental services based on Web technologies, and stimulating new market opportunities ${ }^{3}$. ICT for environmental collaboration is widely recognised as a major step for addressing complex management issues including adaptation to climate change and sustainable management of urban environment.

\footnotetext{
${ }^{1}$ http://ec.europa.eu/environment/seis/, last accessed 11th January 2011

${ }^{2} \mathrm{http}: / /$ www.gmes.info/, last accessed 11th January 2011

${ }^{3}$ http://ec.europa.eu/information_society/digital-agenda/, last accessed 11th January 2011
} 
The European Commission recently funded several projects ${ }^{4}$ in the area of ICT for Sustainable Growth, with a core focus on ICT for Environmental Services and Climate Change aiming at providing the foundations for an infrastructure for monitoring, predicting and managing the environment and its natural resources.

Current research problems addressed by such projects are centred on frameworks, methods, concepts, models, languages and technologies that enable enhanced environmental service infrastructures and platforms (e.g. the ENVISION project [7]). Environmental Information Systems are migrating towards being provided as Software as a Service (SaaS) and will benefit from the utilization and specialization of emerging Infrastructures as a Service (IaaS) and Platforms as a Service (PaaS) as this is emerging under the umbrella of Cloud computing as well as the evolution of the Future Internet ${ }^{5}$ with its three core pillars: an Internet of Services, where applications live in the network, and data becomes an active entity, an Internet of Content, where most of the contents are generated by end-users, and an Internet of Things (IoT), where every electronic device will be an active participant in the network [11]. Cost effective design, development and deployment of environmental models and applications in this context requires novel platforms to emerge, and inter-connect, interoperate and inter-work with each other and with platforms for other verticals. Of particular interest are the architectural foundations of infrastructures and platforms supporting flexible discovery and chaining of distributed environmental services and content, and showing how they combine synergistically to enable better collaborations on the scale required by Future Internet connected environments. This will foster a greater understanding of how open environmental service infrastructures and platforms can enable enhanced collaboration between public authorities, businesses and the general public for a better management of the environment and its natural resources. In this paper, we look at the architectural foundations of open environmental infrastructures and platforms, and propose top-level components of an open architecture (Section 2). We also identify standards relevant to architectures of environmental infrastructures and platforms (Section 3). Standards of ICT for environment are addressed in most detail. We conclude this paper with some challenges (Section 4).

\section{Top-Level Components of the Environmental Monitoring Platform}

The basic architecture of the suggested open environmental platform is depicted in Figure 1. The core platform (three layers in the middle) bridges the gap between the Internet of Things and end-user applications, such as marine monitoring or air quality monitoring and reporting. A relation of the suggested components with the Internet of Services and Internet of Content is depicted in addition. In the remainder of this section, we detail each of these layers (from bottom to the top). Notably, the complete environmental platform may be offered as a service in itself and by this mean implement the Platform as a Service (PaaS) concept.

\footnotetext{
${ }^{4}$ http://cordis.europa.eu/fp7/ict/sustainable-growth/environment_en.html, last accessed 11th January 2011.

${ }^{5} \mathrm{http}: / / \mathrm{www}$. future-internet.eu/
} 


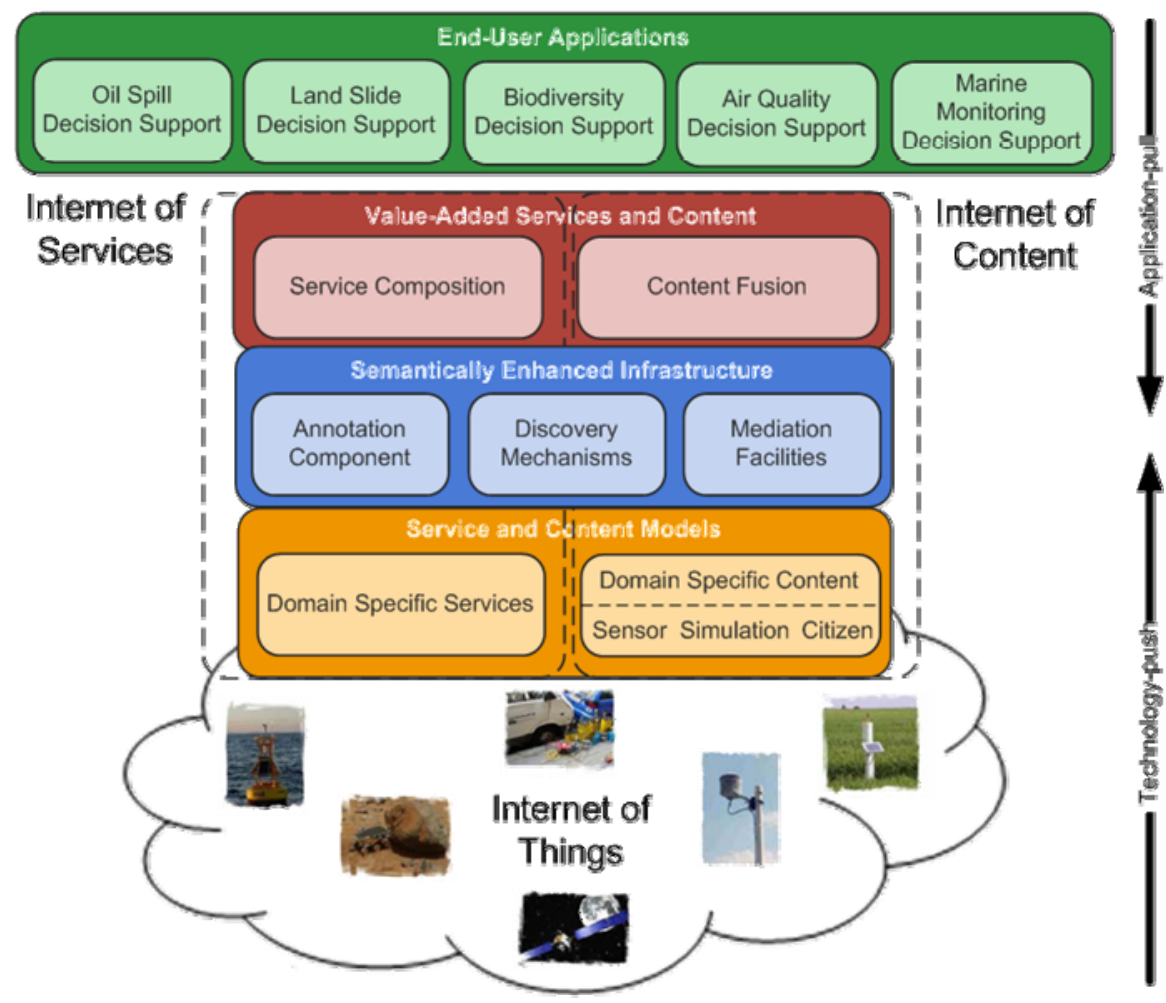

Fig. 1. Top-level Components of the Open Environmental Platform

\subsection{Service and Content Models}

On the bottom level of the platform (Service and Content Models), the diverse resources that are available from the IoT are aggregated into domain specific components, which encapsulate content, as well as access and processing services that are adopted to domain specific needs. We put particular emphasis on the use of open standards and flexibility of the proposed platform. We have to account for new technologies that need to be integrated (technology-push).

Functionalities, non-functional aspects, as well as behaviours of the objects provided by the IoT need to be encapsulated and properly described in order to create a certain level of abstraction on top of which other added-value services can be created. At this level, depending on the types of objects that need to be abstracted, various types of services are created that hide the technicalities and access details of the IoT objects. Examples of such services include sensor services (accessing real-time sensor data well defined interfaces), data services (providing access to relatively static data such as geographical features), or processing services (providing access to geospatial processing, simulations, and other resource-intensive processes). An important challenge here is the development of proper conceptual models for services, that can be customized and extended for different domains. 
In respect to content, we particularly distinguish content based on physical measurements by sensors, content based on environmental simulation/models, and content based on citizen contributed information, especially on Volunteered Geographic information (VGI) [3]. Taking air quality as an example, current values for $\mathrm{CO} 2$ concentration on a particular measurement station are usually measured by physical sensors, simulations result in an areal coverage of $\mathrm{CO} 2$ or in temporal forecasts of the $\mathrm{CO} 2$ concentration, while reports, such as 'it's smelling badly' or 'today it is hard to breath normally in Milan', are part of VGI. For us, this content triangle (Figure 2) serves the basis for all environmental information in the Web. An extension to a rectangle, including literature based content, could be discussed.

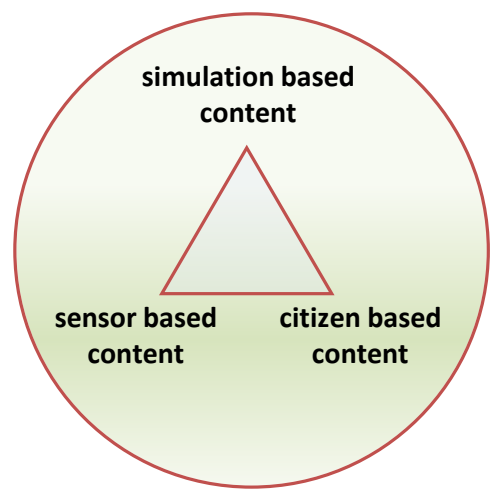

Fig. 2. Triangle of Geospatial Content (adopted from [10])

Specific content models rely on vocabulary generation, conceptual data modelling, and encodings that have to be developed in each specific domain. Inputs from the IoT are wrapped according to each (domain) specific model including required adapters on data types and values. In order to ease interoperability within and across domains, it is desirable to establish a common generic model for integrating content coming from each of the identified kinds of sources (sensor, citizen and simulation) more easily. Nevertheless, the actual integration task is part of the next layer.

\subsection{Semantically Enhanced Infrastructure}

At its intermediate layer in Figure 1 (Semantically Enhanced Infrastructure), the Open Environmental Platform provides harmonized access to the IoT. It facilitates semantic discovery based on annotations. Harmonized access to the IoT is provided in form of mediators that provide encapsulating services.

Semantic annotation should be used to describe the intended interpretation of services and content. Resource descriptions shall make use of existing domain vocabularies and ontologies. This requires (i) tools for creating vocabularies, ontologies and resource descriptions, and (ii) according storage facilities. Discovery mechanisms shall facilitate these descriptions and provide human users and machines with the capabilities to search for desired services or content using their own expressions or a predefined set of domain terms/concepts. For example, users may be provided with 
terms in English, French and German, whereas the initial resources are only described in English. Once a resource or a set of resources has been discovered, users have to be able to retrieve it. This process may include mediation, i.e. the translation of the initial domain model of a provided resource into that of the consumer. Apart from natural language translations, this may include unit conversions or more sophisticated schema mapping rules [12].

\subsection{Value-Added Services and Content}

The third layer of the architecture (Value-Added Services and Content) supports the creation of added value products by offering methodologies and facilities for content fusion and service composition. This layer makes use of the semantic discovery and mediation mechanisms provided by the semantically enhanced infrastructure and to access services or content coming from physical sensors, (environmental) simulations and citizen, as well as contextual information on the targeted added value chain.

The design of service compositions for creation of added-value services on top of atomic services encapsulating the different object provided by the IoT, requires the use a graphical language to allow for user-friendly specification of service chains by domain experts as well as users with little technical skills. The composition language needs to provide a sufficient level of abstraction, while still enabling sufficient level of detail so that the graphical service compositions can be used to generate complete executable service descriptions. The composition language must be able to compose different kinds of services including sensor, data, and processing services, as well as to express control and data flow dependencies between such services. A language based on (a subset of) the emerging Business Process Modelling Notation (BPMN) standard [6], extended with declarative features for flexible modelling of compositions and explicit treatment of data mediation and uncertainty would be a proper candidate for addressing the representational needs of environmental service compositions.

Given the harmonised access to data and the specified context, for example using air quality data for calculating individual exposure indexes, Content Fusion components provide the means for streamlining the available data to a particular use. Sabeur et al. proposed four fusion levels [8]:

- Fusion level 0 provides prepossessing of data, such as smoothing, interpolation, and re-formatting;

- Fusion level 1 offers algorithms for auto-correlation and Fourier analysis and the like, in order to identify objects or trends in the incoming data;

- Fusion level 2 includes phenomenology models for estimating and refining previous fusion results; and

- Fusion level 3 provides prediction and correction information, for example based on Kalman filtering.

The value added content can be exposed after each of the fusion steps and may serve input to multiple further processing steps. In this way, it provides manifold contributions to the Internet of Content. Notably, the modelling and propagation of uncertainties should be considered in the complete fusion process. This will require a quantification of initial errors and vagueness in the raw data coming from the lower levels of the proposed platform. 


\subsection{End-User Applications}

Complementary to the technology-push, novel developments have to account for arising user requirements (application-pull). Otherwise, implementing sophisticated technology solutions, which are not used in the mid- and long-term, might be exciting, but remain a unprofitable exercise. In the following we outline a subset of the manifold applications, which should be supported by open environmental platforms. Their specific technical and non-technical requirements will help to sharpen the specifications of the components on the lower layers of the proposed solution and thereby to develop a flexible solution for information systems that account for all environmental spheres, including the atmospheric, terrestrial and marine domains.

Ground instabilities caused by mud flows, landslides or ground failure may cause significant physical and socio-economic damage in many regions of the world. Being able to correctly forecast such phenomena for proper prevention and mitigation implies the availability of and access to relevant environmental models, data, and services, and the ability of combining them in meaningful ways. Issues such as finding a landslide risk model with specific characteristics, which produces the required output data that fits given input data, chaining models for creating value added services such as landslide risk assessment models and making them accessible for the wider community pose great challenges for discovery and composition of both services and content.

Biodiversity related applications add user-contributed content as an important aspect to data acquisition. While many environmental monitoring applications reply on classical sensors, and environmental simulation and modelling, the biodiversity community retrieves large amounts of information from volunteers, which post their observations on species occurrences on diverse networking platforms. Birdwatch ${ }^{6}$ provides one of the many impressive examples, in this case related to the occurrence of birds. The handling, quality assurance, and integration of such information is public authority data, such as protected sites, particularly challenges content fusion and semantic content descriptions.

Among others, air quality monitoring bear the challenge of assessing individualized exposure. Today, we have easy access to a great deal of information via television, radio and the World Wide Web. This includes pollution, pollen and meteorological data which are all relatively easily accessed in one or more dissemination channels. All this data contributes to a common sense, but it is not tailored to individual user needs. Relevancy of data and interpreting it are key issues for users today, especially with regards to pollen and pollution. The envisioned platform shall therefore aid the users in tailoring information relevant to their individual requirements, which again poses requirements on the functionalities for implementing the added value chain from general purpose to individualised information.

For the marine domain, the challenge is to create synergies with the market and with policy needs that are necessary to deliver significant value added to Europe from its vast marine resources. Enabling technology platforms are currently deployed across a range of existing marine related sectors including shipping, security and logistics, environmental monitoring and offshore energy. Next generation platforms

\footnotetext{
${ }^{6}$ http://www.birdwatch.co.uk/
} 
have to dissolve national borders. They shall address these developments in respect to distributed sensing, and wireless and cable communications. In the marine domain, oil spills are an extremely sensitive topic. Accidental oil releases to the sea may have severe environmental, social and economic consequences, with vital natural resources and human enterprises being at risk. For oil spill decision making, it is essential to be able to predict the fate and effects of the spilled oil. Fate prediction requires data on the spill (location, time, amount, oil type), the environmental conditions (wind, current), and geography (sea depths, coast line). Effects prediction is based on data about natural resources (e.g. fish populations). Being able to combine such data (possibly made available through service) in real-time for prediction of the fate of the oil spill implies sophisticated discovery, composition, and integration mechanisms.

\section{Relevant Standards}

Open standards play a key role for interoperability between emerging environmental platforms. By only briefly pointing to standards etc. which have to be considered at the surrounding levels, we particularly focus on required standards on ICT for environment.

Depending on the exact field, end-user applications have to account for a large series of legal documents and standards. The UN Convention on Biodiversity, Clean Air for Europe, and the Marine Strategy Framework Directive are only some examples of these European and global Directives, Regulations and Strategies effecting particular environmental thematic areas. In total several hundred of similar texts apply to environmental monitoring on European and national level.

Value added services and content partially involve mainstream ICT standards, such as Business Process Modelling Notation (BPMN) [6] or Business Process Execution Language (BPEL) [5] for service composition. However, special standards and best practises exist for the environmental (and wider geospatial) community. Most notably, these include:

○ ISO 19119 (Geographic information - Services) [4] is a standard defined by the Technical Committee 211 (TC211) of the International Standardisation Organization (ISO). It defines the architecture patterns for service interfaces used for geographic information and presents a geographic services taxonomy which includes human interaction services, model/information management services, geographic processing services (spatial, thematic, temporal) and communication services. The standard includes guidelines for the selection and specification of geographic services from both platformneutral and platform-specific perspectives. In fact, the various types of services proposed by this standard are relevant for all layers in Figure 1 where spatiotemporal aspects are relevant.

- The Sensor Web Enablement (SWE) of the Open Geospatial Consortium (OGC) [1] provides standardized interfaces and metadata encodings that aim to enable real time integration of heterogeneous sensor webs into the information infrastructure. Sensor Model Language (SensorML), Observations \& Measurements (O\&M), Sensor Observations Service (SOS) are the most important specifications provided by SWE. These standards become highly 
relevant in relation to value added content and services. SWE standards currently undergo a phase of broad acceptance.

- The Worl Wide Web Consortium (W3C) Semantic Sensor Network Incubator Group $^{7}$ developed ontologies that define the capabilities of sensors and sensor networks, and provides semantic annotations of a key language used by services based sensor networks. On top of its Semantic Web standards (RDF, OWL), W3C is setting the standards foundations for semantic interoperability (see also below).

The Semantically Enhanced Infrastructure layer serves the building blocks to the higher-level value-added components. Here, standards of the W3C Semantic Web community, such as the Resource Description Framework (RDF) ${ }^{8}$ and the Web Ontology Language (OWL) ${ }^{9}$ should be applied.

\section{Challenges and Outlook}

The realization of the desired open platform faces several challenges. For us, these include (ordered from the bottom layer of the platform to the top):

- Interoperability in a heterogeneous environment: Dealing with resource discovery, processing support, service interfacing, and intermodal operation between heterogeneous types of resources. All of the above required semantically rich and extendible data models.

- Advanced data mining: Including the handling of large volumes of data, pattern recognition depending on context, and data flow processing.

- Data fusion with uncertainty analysis: Involving uncertainty and the combination of data and processing, but also the economic value of information from source to consumer. Security mechanisms and error-propagation are some of the required tools.

- Dynamic service composition: Considering run-time identification of suitable service components, their bindings and execution.

o User support: Covering generation of templates for end-user applications, best practices for application development and training.

As many prototype solutions already exist, issues of scalability, robustness, and quality of service are central to all of the listed issues [9]. An integrated approach, which is easy to instantiate, remains to be developed. The required balance between application-pull and technology-push can only be reached if the gap between platform developers and intended users can be bridged. This aspect is the most crucial for successful implementation.

We are currently addressing these issues within numerous de- (or loosely) coupled research projects. The Europe 2020 strategy $^{10}$ and two of its seven flagship initiatives (the Digital Agenda and Innovation Union) provide possibilities for long term funding.

\footnotetext{
${ }^{7}$ http://www.w3.org/2005/Incubator/ssn/wiki/Main_Page, last accessed 11th January 2011.

${ }^{8}$ http://www.w3.org/RDF/ http://www.w3.org/RDF/

${ }^{9} \mathrm{http} / / / \mathrm{www} . \mathrm{w} 3$. org/TR/owl2-overview/

${ }^{10} \mathrm{http}: / / \mathrm{www}$. europe2020.org/?lang=en, last accessed 11th January 2011.
} 
Acknowledgements. The presented research has been partially funded by the ENVIROFI FP7 project (FP7-2011-ICT-FI-284898) and ENVISION project (249120).

\section{References}

1. Botts, M., Percivall, G., Reed, C., Davidson, J.: OGC® Sensor Web Enablement: Overview and High Level Architecture. In: Nittel, S., Labrinidis, A., Stefanidis, A. (eds.) GSN 2006. LNCS, vol. 4540, pp. 175-190. Springer, Heidelberg (2008)

2. European Parliament and Council. Directive 2007/2/EC of the European Parliament and of the Council of 14 March 2007 establishing an Infrastructure for Spatial Information in the European Community (INSPIRE). Official Journal on the European Parliament and of the Council (2007)

3. Goodchild, M.: Citizens as sensors: the world of volunteered geography. GeoJournal 69(4), 211-221 (2007)

4. ISO/TC211. 19119 Geographic Information - Services. ISO/TC211 Standards (2005)

5. OASIS, Web Services Business Process Execution Language Version 2.0. Committee Specification. OASIS WS-BPEL TC (2007)

6. OMG. Business Process Modeling Notation (BPMN) Version Beta 1 for version 2.0 OMG Document Number: dtc/2009-08-14 (August 2009)

7. Roman, D., Schade, S., Berre, A.J., Bodsberg, N.R., Langlois, J.: Environmental Services Infrastructure with Ontologies - A Decision Support Framework. In: 23rd International Conference on Informatics for Environmental Protection (EnviroInfo 2009), Berlin, September 9-11, pp. 287-295. Shaker Verlag, Aachen (2009)

8. Sabeur, Z., Middleton, S., Veres, G., Zlatev, Z., Salvo, N.: Generic Sensor Data Fusion Services for Web-enabled Environmental Risk Management and Decision-Support Systems. In: European Geophysics Union, General Union Assembly, Vienna (May 2010)

9. Schade, S.: A Sensor Web for the Environment in Europe. In: Lemke, M. (ed.) 2nd Usage Area Workshop: Future Internet Initiative, Brussels, Belgium, June 21-22 (2010)

10. Schade, S., Craglia, M.: A Future Sensor Web for the Environment in Europe. In: EnviroInfo 2010, Cologne/Bonn, Germany (2010)

11. Vermesan, O., et al.: Internet of Things Strategic Research Roadmap. CERP-IoT 2009, Report Available at http: / /ec.europa.eu/information_society/ policy/rfid/documents/in_cerp.pdf

12. Wache, H., Vögele, T., Visser, U., Stuckenschmidt, H., Schuster, G., Neumann, H., Hübner, S.: Ontology-Based Integration of Information - A Survey of Existing Approaches. In: IJCAI 2001 Workshop: Ontologies and Information Sharing, Seattle, WA (2001) 